\title{
Dipeptidyl peptidase 4 inhibitor linagliptin can decrease the dosage of erythropoiesis- stimulating agents in patients on hemodialysis
}

\author{
Masaki Aono ${ }^{*}$ (D) and Yuzuru Sato
}

\begin{abstract}
Background: Dipeptidyl peptidase 4 (DPP4) inhibitors are very useful for the treatment of type 2 diabetes, especially to patients undergoing hemodialysis (HD). The main action of DPP4 inhibitors is to increase the concentration of incretin which stimulate the insulin secretion from pancreatic beta cells. Recently, the extra-pancreatic actions of DPP4 inhibitors were suggested by many reports; a study has shown that hematopoiesis in mice after radiation or chemotherapy was enhanced receiving an oral DPP4 inhibitor sitagliptin. It suggests the possibility that DPP4 inhibitors can reduce the dosage of erythropoiesis-stimulating agents (ESAs) in HD patients. But there is no clinical report about the effect of DPP4 inhibitors on renal anemia in HD patients. This study was designed to confirm the clinical effect of DPP4 inhibitors on the reduction of dosage of ESA or improvement of renal anemia.
\end{abstract}

Methods: Over a 6-month period from October 2013 to June 2014, a daily dose of DPP4 inhibitor linagliptin (5 mg/ day) was administered orally to 25 patients on HD who were diagnosed with diabetes mellitus in our institution. The dosage of ESA darbepoetin alfa (DA), ESA-resistant index (ERI: DA dosage/hemoglobin), hemoglobin concentration, transferring saturation (TSAT), ferritin, and high-sensitivity C-reactive protein (hs-CRP) were measured and averaged for 3 months before and after the treatment.

Results: At the end of the 6 months, 12 patients were excluded from the analysis due to death, hospitalization in another hospital, cancer, or withdrawal. After administration of linagliptin, the dosage of DA and ERI decreased significantly $(P<0.05$, paired $t$ test).The dosage of DA decreased from a mean \pm SD of $23.9 \pm 21.2$ to $11.3 \pm 9.7$ $(\mu \mathrm{g} /$ week), and ERI decreased from $2.3 \pm 2.1$ to $1.0 \pm 0.9$. Hemoglobin concentration, white blood cell (WBC) count, platelet count, ferritin, and TSAT level were not different. Hs-CRP, as the marker of inflammation, was not different, too.

Conclusions: This study suggests that DPP4 inhibitor linagliptin can decrease the dosage of ESA without the influence of iron metabolism and inflammation. The previous study has shown that DPP4 cleaves within N-terminal of erythropoietin and decreases their activity in mice. We speculate that DPP4 inhibitors can increase the concentration of erythropoietin by inhibiting this action of DPP4.

\section{Background}

Dipeptidyl peptidase 4 (DPP4) inhibitors are very useful for the treatment of patients with type 2 diabetes mellitus (T2DM), especially those undergoing hemodialysis in Japan. Other glucose-lowering agents, such as sulfonylureas, biguanides, thiazolidinediones, and nateglinide (an ultrafast-acting insulin

* Correspondence: masaki-aono@satohp.co.jp

Internal Medicine, Sato Junkanki Hospital, Matsuyama, Japan secretagogue), are contraindicated in dialysis patients in Japan [1].

DPP4 inhibitors selectively block DPP4, increase the activity of glucagon-like peptide-1 (GLP-1), stimulate insulin secretion, and inhibit glucagon secretion. Recently, the extra-pancreatic actions of DPP4 inhibitors have been indicated by many reports, including kidney and cardiovascular protection, among other effects [2-5].

DPP4 is a protease that cleaves to selected amino acids in the penultimate $\mathrm{N}$-terminal position and has the 
potential to alter protein function [6]. Reportedly, DPP4 cleaves to the $\mathrm{N}$-termini of factors such as the colony-stimulating factor (CSF), granulocyte-macrophage (GM)-CSF, G-CSF, interleukin-3, and erythropoietin and can decrease their activity [7]. Both in vitro and in vivo, DPP4 knockout or DPP4 inhibition enhanced CSF activities [7]. In mice undergoing radiation or chemotherapy, hematopoiesis was enhanced after receiving the oral DPP4 inhibitor sitagliptin. That study suggested that DPP4 inhibitors can reduce the dosage of erythropoiesisstimulating agents (ESAs) required for patients undergoing hemodialysis. However, presently, no clinical reports have assessed the effect of DPP4 inhibitors on renal anemia in patients with T2DM undergoing hemodialysis. The aim of our study was to evaluate whether DPP4 inhibitors reduced ESA dosage or contributed to the improvement of renal anemia in Japanese patients with T2DM undergoing hemodialysis.

\section{Methods}

\section{Study design and participants}

The present study was a cohort study including patients with T2DM undergoing hemodialysis at the Sato Junkanki Hospital in Matsuyama, Japan, between October 2013 and June 2014. We divided the patients into two groups for comparison: one group received DPP4 inhibitor (DPP4+ group) and the other did not receive DPP4 inhibitor (DPP4- group).

A daily dose of DPP4 inhibitor linagliptin ( $5 \mathrm{mg} /$ day) was administered orally to 25 patients diagnosed with T2DM on hemodialysis (DPP4+ group). At the end of the 6 months of treatment, 12 patients were excluded from the analysis because of death, transfer to another hospital, cancer, or withdrawal. Thus, data of 13 patients in the DPP4+ group were included in the analysis. A total of 16 patients were only treated by subcutaneous insulin injection and did not receive DPP4 inhibitor linagliptin (DPP4- group). As a result, a total number of 29 patients were included in the analysis.

The administration of linagliptin was decided case-bycase, as there are currently no clear criteria for the use of linagliptin. The only treatments administered in this study were linagliptin as a DPP4 inhibitor and darbepoetin alfa (DA) as an ESA, except for the treatment of DM.

The study was approved by the Ethics Committees of Sato Junkanki Hospital. All the participants gave written informed consent.

\section{Clinical measures}

Clinical measures obtained before and after treatment included age, sex, hemodialysis duration, hemoglobin, transferring saturation (TSAT), ferritin, high-sensitivity C-reactive protein (hs-CRP), and serum glycated albumin. The dosage of ESA DA, ESA-resistant index (ERI:
DA dosage/hemoglobin), hemoglobin concentration, TSAT, ferritin, and hs-CRP were measured and averaged 3 months before and 3 months after the treatment.

The dosage of DA was adjusted to maintain a hemoglobin concentration between 10 and $12 \mathrm{~g} / \mathrm{dl}$ according to the guidelines of the Japanese Society for Dialysis Therapy.

\section{Statistical analysis}

Statistical analyses of data were performed using Statistical Package for the Social Sciences (SPSS Inc., Chicago, IL, USA) for Windows version 17.0. Mann-Whitney $U$ test was used to compare the demographic characteristics among the two groups because not all quantitative parameters have normal distributions, according to the Shapiro-Wilk test. We used a paired Wilcoxon signedrank test within the groups. The results are expressed as mean \pm standard deviation (SD). Statistical significance was set at $P<0.05$.

\section{Results}

There were no significant differences between the two groups with respect to baseline demographic characteristics, except for the dosage of DA and ERI, which were higher in the DPP4+ group before treatment as shown in Table 1. However, these differences were not significantly different $(P>0.05)$.

After administration of linagliptin, the dosage of DA and ERI decreased significantly $(P<0.05$, Wilcoxon signed-rank test). The dosage of DA decreased from a mean $( \pm \mathrm{SD})$ of $23.9 \pm 21.2$ to $11.3 \pm 9.7$ ( $\mu \mathrm{g} /$ week), and ERI decreased from $2.3 \pm 2.1$ to $1.0 \pm 0.9$ (Table 2).

Table 1 Demographic characteristics before treatment

\begin{tabular}{llll}
\hline & DPP4+ & DPP4- & $P$ \\
\hline Age & 13 & 16 & \\
Male & $70.5 \pm 12.4$ & $71.7 \pm 10.2$ & NS \\
BMl & 8 & 11 & NS \\
HD duration & $21.7 \pm 3.1$ & $22.6 \pm 4.1$ & NS \\
Hb & $6.5 \pm 5.5$ & $10.4 \pm 6.2$ & NS \\
Dosage of DA & $10.8 \pm 0.6$ & $11.0 \pm 0.8$ & NS \\
ERI & $23.9 \pm 21.2$ & $16.1 \pm 12.1$ & NS \\
Albumin & $2.28 \pm 2.08$ & $1.52 \pm 1.19$ & NS \\
TSAT & $3.6 \pm 0.4$ & $3.5 \pm 0.3$ & NS \\
Ferritin & $24.2 \pm 6.6$ & $28.9 \pm 13.5$ & NS \\
hs-CRP & $264.7 \pm 334.6$ & $138.8 \pm 137.3$ & NS \\
GA & $5335 \pm 6494$ & $4345 \pm 9372$ & NS \\
\hline DAdabepoetin & $22.1 \pm 5.8$ & $25.2 \pm 5.8$ & NS \\
\hline
\end{tabular}

$D A$ darbepoetin alfa, $E R I$ erythropoiesis-stimulating agent-resistant index, $H b$ hemoglobin, TSAT transferrin saturation, BMI body mass index, GA glycated albumin 
Table 2 Change of parameters

\begin{tabular}{lccc}
\hline & Before treatment & Post treatment & Wilcoxon signed-rank test \\
\hline Dosage of DA & $23.9 \pm 21.2$ & $11.3 \pm 9.7$ & $P<0.05$ \\
ERI & $2.27 \pm 2.08$ & $1.04 \pm 0.90$ & $P<0.05$ \\
$\mathrm{Hb}$ & $10.8 \pm 0.6$ & $11.4 \pm 0.9$ & $\mathrm{NS}$ \\
hs-CRP & $5335 \pm 6495$ & $2568 \pm 4059$ & $\mathrm{NS}$ \\
TSAT & $24.2 \pm 6.5$ & $31.9 \pm 11.0$ & $\mathrm{NS}$ \\
Ferritin & $264.7 \pm 334.6$ & $249.8 \pm 224.2$ & $\mathrm{NS}$ \\
Albumin & $3.6 \pm 0.4$ & $3.6 \pm 0.3$ & NS \\
WBC & $5789 \pm 1282$ & $5687 \pm 2121$ & NS \\
Plt & $15.6 \pm 6.4$ & $15.2 \pm 6.1$ & NS \\
\hline
\end{tabular}

$D A$ darbepoetin alfa, $E R I$ erythropoiesis-stimulating agent-resistant index, $H b$ hemoglobin, $T S A T$ transferrin saturation, WBC white blood cells count, Plt platelet count

Hemoglobin concentration, white blood cell (WBC) count, platelet count, ferritin, and TSAT level were not significantly different. There were no differences in HsCRP level.

In contrast to the DPP4+ group, the dosage of DA and ERI in the DPP4- group did not decrease significantly after treatment with insulin (Figs. 1 and 2).

\section{Discussion}

DPP4 inhibitors selectively block DPP4, increase the activity of glucagon-like peptide-1 (GLP-1), stimulate insulin secretion, and inhibit glucagon secretion. The sole use of a DPP4 inhibitor is considered to rarely induce hypoglycemia because its plasma glucose-lowering effect is dependent on the plasma glucose level. Additionally, DPP4 inhibitors improve glycemic control without increasing body weight [1]. Based on these characteristics, a DPP4 inhibitor is very useful for diabetic patients on hemodialysis. Linagliptin is mainly excreted from the body unchanged via fecal and renal excretion [8]. Janet et al. reported that in patients with T2DM and severe renal impairment, linagliptin provided clinically meaningful improvements in glycemic control with very low risk of severe hypoglycemia, stable body weight, and no drugrelated renal failure [9]. Further, it has been reported that linagliptin can be used at a regular dose in patients on hemodialysis [10].

Recently the extra-pancreatic actions of DPP4 inhibitors were indicated by many reports, including kidney and cardiovascular protection, among other effects [25]. Our study showed that DPP4 inhibitor linagliptin significantly reduced the dosage of DA for T2DM patients on hemodialysis with renal anemia and significantly decreased ERI as well.

The resistance to ESAs might be caused by inflammation, which has a negative effect on erythropoietin receptors [11]. In our study hs-CRP, a marker of inflammation, did not change significantly before or after

\section{Dosage of DA}
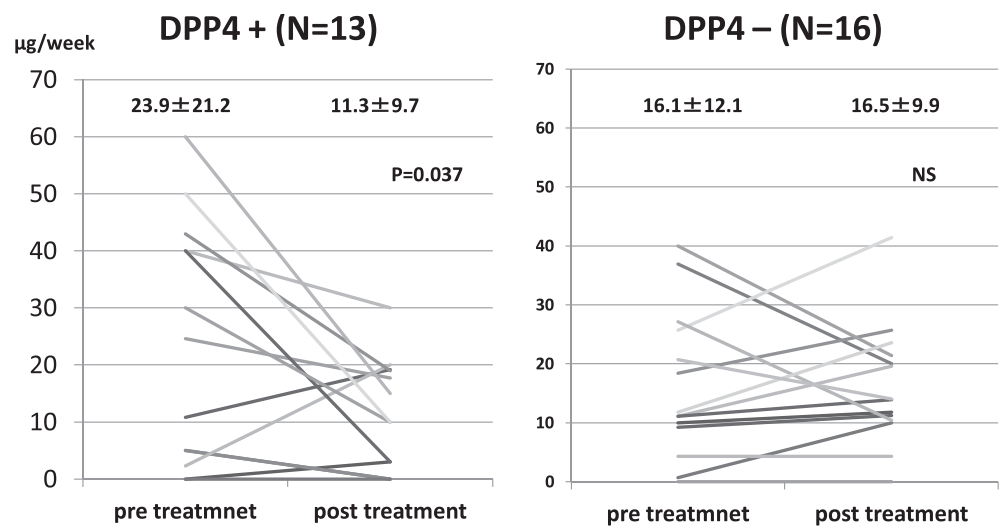

DA:darbepoetin alfa, DPP4:Dipeptidyl peptidase 4

Fig. 1 Change in dosage of darbepoetin alfa 


\section{ERI}

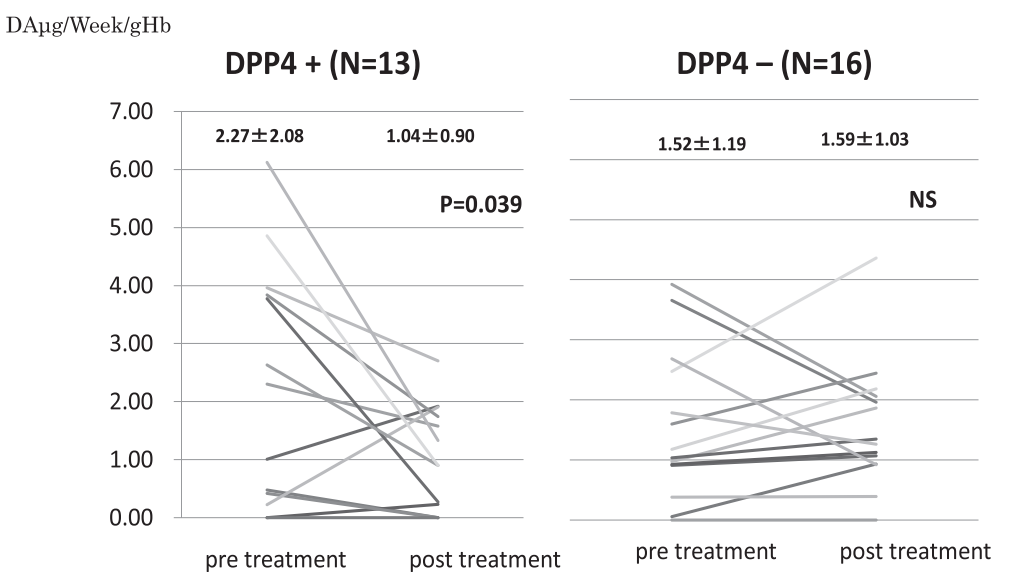

ERI: erythropoiesis-stimulating agents resistant index

Fig. 2 Change in ESA-resistant index

treatment. Additionally, insufficient iron metabolism can cause erythropoietin-resistant anemia. However, we also observed that TSAT and ferritin remained unchanged throughout the treatment. Thus, we concluded that DPP4 inhibitor linagliptin can reduce the dosage of ESA without affecting iron metabolism or inflammation.

A previous study by Broxmeyer showed that DPP4 cleaved within the N-termini of the CSFs, GM-CSF, GCSF, interleukin-3, and erythropoietin and decreased their activity. Dpp4 knockout or DPP4 inhibition enhanced CSF activities both in vitro and in vivo. The reduced activity of DPP4-truncated versus full-length human GM-CSF was mechanistically linked to effects on receptor-binding affinity, induction of GM-CSF receptor oligomerization, and signaling capacity. Hematopoiesis in mice after radiation or chemotherapy was enhanced in Dpp4-/- mice or mice receiving an orally active DPP4 inhibitor. DPP4 inhibition enhanced engraftment in mice without compromising hematopoietic stem cell function, suggesting the potential clinical utility of this approach [7].

Heather O'Leary et al. [6] suggested that DPP4 is a protease that cleaves selected amino acids at the penultimate $\mathrm{N}$-terminal position and has the potential to alter protein function. Recent reports on the number of proteins that have DPP4 sites, and on how DPP4 truncation may alter hematopoiesis based on the full protein length versus the truncated state, have shown that DPP4 truncation of CSFs alters their function and that the activity of these CSFs can be enhanced when DPP4 activity is inhibited. Thus, we speculate that the DPP4 inhibitor can increase the concentration of erythropoietin by inhibiting the action of DPP4 on erythropoietin.
In this study, the number of peripheral blood cells (WBC, platelet) did not increase, because blood cells tend to remain unchanged in hemodialysis patients. The level of G-CSFs did not decrease either. Thus, we presume that this was caused by the effect of the DPP4 inhibitor on erythropoietin. However, we did not measure the serum erythropoietin concentration because erythropoietin was administrated to patients at the hemodialysis session. Thus, we have merely speculated on the possible mechanism behind the effect of DPP4 inhibitor on erythropoietin.

Terawaki et al. reported that linagliptin can decrease serum levels of oxidized low-density lipoprotein in patients with T2DM undergoing hemodialysis independent of its glucose-lowering effect [3]. Additionally, the GLP1 receptor agonist liraglutide has been demonstrated to decrease oxidative stress independent of its glucoselowering effect [12]. We presume that as a result of the reduction of oxidative stress, red blood cell survival may have increased, thereby improving the ERI in our study.

Our study has some important limitations. This was merely a pilot study, and thus, our study population was small and the observation period was short. The patients were not randomized to treatment, and there was no blinding in place, which limits the strength of our results, but we found an association between the DPP4 inhibitor and erythropoietin. Confirmation of our findings is required in a large-cohort, randomized, double-blind study.

\section{Conclusions}

Based on the preliminary findings of the present study, DPP4 inhibitors could be beneficial for patients with T2DM undergoing hemodialysis. We conclude that the DPP4 inhibitor linagliptin can reduce the dosage of ESA in patients undergoing hemodialysis. 


\section{Acknowledgements}

The data were obtained with permission from Sato Junkanki Hospital.

All authors received no funding from anyone.

\section{Authors' contributions}

YS conceived of the study and participated in its design and coordination and helped to draft the manuscript. Both authors read and approved the final manuscript.

\section{Competing interests}

The authors declare that they have no competing interests.

Received: 21 October 2015 Accepted: 21 July 2016

Published online: 28 September 2016

\section{References}

1. Nakao T, Inaba M, Abe M, Kaizu K, Shima K, Babazono T, Tomo T, Hirakata T. Best practice for diabetic patients on hemodialysis 2012. Ther Apher Dial. 2015;19:40-66.

2. Fisman EZ, Tenenbaum A. Antidiabetic treatment with gliptins: focus on cardiovascular effects and outcomes. Cardiovasc Diabetol. 2015;14:15-10265. 129 Page 1 of 13.

3. Terawaki $Y$, Nomiyama T et al. Efficacy of dipeptidyl peptidase-4 inhibitor linagliptin in patients with type 2 diabetes undergoing hemodialysis. Diabetol Metab Syndr. 2015:7:44. P15-05456.

4. Shi S, Srivastava SP, et al. Interactions of DPP-4 and integrin $\beta 1$ influences endothelial-to-mesenchymal transition. Kidney Int. 2015;88:479-89.

5. Keizo K,1, Sen S, et al. Linagliptin-mediated DPP-4 inhibition ameliorates kidney fibrosis in streptozotocin-induced diabetic mice by inhibiting endothelial-to-mesenchymal transition in a therapeutic regimen. Diabetes. 2014:63:2120-31.

6. Heather $\mathrm{O}$, Xuan $\mathrm{O}, \mathrm{Hal}$ EB, et al. The role of dipeptidyl peptidase 4 in hematopoiesis and transplantation. Curr Opin Hematol. 2013;20:314-9.

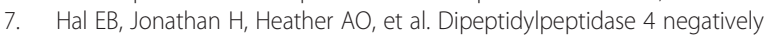
regulates colony-stimulating factor activity and stress hematopoiesis. Nat Med. 2012;18:1786-96.

8. Blech S, Ludwig-Schwellinger $\mathrm{E}_{\text {, et }}$ al. The metabolism and disposition of the oral dipeptidyl peptidase-4 inhibitor, linagliptin, in humans. DMD. 2010; 38:667-78.

9. McGill J B, Sloan M, et al. Long-term efficacy and safety of linagliptin in patients with type 2 diabetes and severe renal impairment. Diabetes Care. 2013;36(2):237-44

10. Graefe-Mody U, Friedrich C. Effect of renal impairment on the pharmacokinetics of the dipeptidyl peptidase-4 inhibitor linagliptin. Obes Metab. 2011;13:939-46.

11. Karien VP. Mechanisms of disease: erythropoietin resistance in patients with both heart and kidney failure. Nat Clin Pract Nephrol. 2008;4:47-57.

12. Hendarto H, Inoguchi T, Maeda Y, Ikeda N, Zheng J, Takei R, et al. GLP-1 analog liraglutide protects against oxidative stress and albuminuria in streptozotocin-induced diabetic rats via protein kinase A-mediated inhibition of renal NAD(P)H oxidases. Metabolism. 2012;61:1422-34.

\section{Submit your next manuscript to BioMed Central and we will help you at every step:}

- We accept pre-submission inquiries

- Our selector tool helps you to find the most relevant journal

- We provide round the clock customer support

- Convenient online submission

- Thorough peer review

- Inclusion in PubMed and all major indexing services

- Maximum visibility for your research

Submit your manuscript at www.biomedcentral.com/submit 